POS $\quad$ PROCEEDINGS

\title{
Electromagnetic processes in ultra-peripheral lead-lead collisions with ATLAS
}

\author{
Marcin Guzik, on behalf of the ATLAS Collaboration \\ AGH University of Science and Technology \\ Faculty of Physics and Applied Computer Science \\ Al. Mickiewicza 30, 30-059 Cracow, Poland \\ E-mail: marcin.guzikecern.ch
}

\begin{abstract}
The large equivalent-photon fluxes accompanying $\mathrm{Pb}$ ion beams at the LHC initiate photonphoton and photo-nuclear interactions which dominate when the colliding nuclei have large impact parameter (ultra-peripheral collisions). These electromagnetically-induced processes are sensitive to the nuclear wave-function and in particular the nuclear modifications of the nucleon parton distribution functions (nPDFs). As such, they are complementary to the ongoing $\mathrm{p}+\mathrm{A}$ program at RHIC and the LHC, as well as the upcoming electron-ion collider (EIC) program in the US. High-mass dilepton pair continuum rates have been measured and compared with theoretical predictions to test expectations for two-photon interactions, and good agreement with model calculations is obtained. Evidence for the elastic scattering of photons $\gamma \gamma \rightarrow \gamma \gamma$ ("light-bylight" scattering) will be presented, a previously unobserved process made possible by the high photon flux and low event pileup provided by the LHC. While of intrinsic interest as a heretoforeunobserved standard model process, it has also been proposed as a clean channel for searches for beyond the standard model (BSM) physics.
\end{abstract}

XXV International Workshop on Deep-Inelastic Scattering and Related Subjects

3-7 April 2017

University of Birmingham, $U K$ 


\section{Introduction}

The data from the collisions of ultrarelativistic lead nuclei can be explored not only in the regime in which they are interacting hadronically producing dense nuclear matter known as the quark-gluon plasma but also when the impact parameter of the collision $b$ (see Figure 1) is much larger than the sum of the radii of the colliding particles favouring the purely electromagnetic (EM) interactions. In the second scenario, known under a term of Ultra-Peripheral Collisions (UPC), the strong EM fields surrounding the nuclei can be thought of as the fluxes of quasi-real photons and the EM reaction itself can be treated as the photon-photon interaction. Such an approach proposed for the first time by Fermi [1] is known as the Weizsacker-Williams [2, 3] or Equivalent Photon Approximation (EPA).

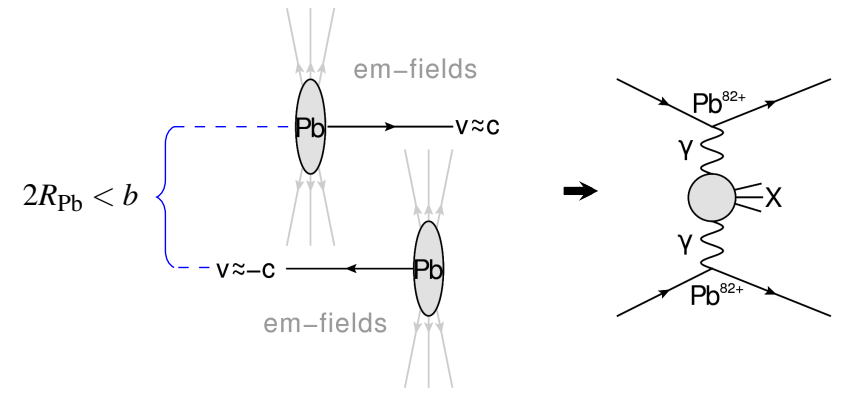

Figure 1: The ultra-peripheral collision of two lead ions. The electromagnetic interaction between the ions can be described as photon-photon interaction producing final state $X$.

Within the framework of this approximation, it is possible to express the cross-section of (quasi)electromagnetic interaction as the integral of the elementary QED cross-section weighted by the number of photons of energy $\omega$ denoted here as $n(\omega)$

$$
\sigma_{A_{1} A_{2}(\gamma \gamma) \rightarrow A_{1} A_{2} X}^{E P A}=\iint d \omega_{1} d \omega_{2} n_{1}\left(\omega_{1}\right) n_{2}\left(\omega_{2}\right) \sigma_{\gamma \gamma \rightarrow X}\left(W_{\gamma \gamma}\right),
$$

where the latter can be obtained by the integration of charge form factors of the ions

$$
n(b, \omega)=\frac{Z^{2} \alpha_{e m}}{\pi \omega}\left|\int d q_{\perp} q_{\perp}^{2} \frac{F\left(Q^{2}\right)}{Q^{2}} J_{1}\left(b q_{\perp}\right)\right|^{2} .
$$

The EPA photons are quasi-real having $Q^{2}<\frac{1}{R_{\mathrm{Pb}}^{2}} \approx 10^{-3} \mathrm{GeV}^{2}$ and their maximal energy is of the order of Lorentz factor of the ion over its radius $\omega_{\max } \approx \frac{\gamma}{R}$. The fact that the photon fluxes scale with $Z^{2}$ leads to the coherent enhancement of the EM cross-section of the order of $\sim 10^{7}$ what makes the lead-lead $(\mathrm{Pb}+\mathrm{Pb})$ collisions more attractive from the perspective of EM processes than the $p p$ collisions.

Having all this in mind the Large Hadron Collider (LHC) can be treated as the photon-photon collider providing the data from the UPC collisions to study the photon-photon interactions. In these proceedings the ATLAS results of the measurement of the production of high-mass dimuon pairs and of the search of photon-photon scattering extracted from the $\mathrm{Pb}+\mathrm{Pb}$ data taken in 2015 at the center-of-mass energy per nucleon pair of $5.02 \mathrm{TeV}$ will be presented. The total luminosity of this data amounts to $0.5 \mathrm{nb}^{-1}$. 


\section{Measurement of high-mass dimuon pairs}

The first measurement reported here is the production of high-mass dimuon pairs in UPC $\mathrm{Pb}+\mathrm{Pb}$ collisions $\left(\gamma \gamma \rightarrow \mu^{+} \mu^{-}\right)$by ATLAS [5]. The data for this measurement were selected by the trigger comprising the requirement of at least one loose muon at level 1 , at least one $200 \mathrm{MeV}$ track at HLT, the cut on total transverse energy in the calorimeter below $50 \mathrm{GeV}$ and the requirement of no more than one hit in the inner arrays of the Minimum Bias Trigger Scintillators (MBTS's).

The offline selection consisted of the requirement of two good quality opposite sign muons with $p_{\mathrm{T}}^{\mu}>4 \mathrm{GeV}$ and $\left|\eta^{\mu}\right|<2.4$ with the mass of the pair $m^{\mu \mu}$ greater than $10 \mathrm{GeV}$ and with the requirement of the vertex with no additional tracks.

Signal was modelled with MC generator STARlight 1.1 [6] which is incorporating the leading order QED calculations. The total 12069 events were selected and corrected for the trigger efficiency obtained using the data with two different methods: the tag and probe technique with the cut on pair $p_{\mathrm{T}}$ of $500 \mathrm{MeV}$, used as an equivalent of the mass cut, or an alternative approach with independent minimum bias triggers. They were also corrected for the muon reconstruction and identification efficiency, derived from single muon MC, and for vertex efficiency.

After applying those corrections the small excess of data over MC was observed in the tail of the acoplanarity distribution (Aco $=(1-|\Delta \phi| / \pi)$, see Figure 2). The two assumptions about its origin were tested. The first was that the excess could be attributed to the background in which case it was approximated by the power law fit and subtracted from the data. In the second approach, it was treated as the signal, that may have potentially been caused by the higher-order effects not included in the STARlight simulation, and kept. Mean of the two obtained in such a way results was taken as the nominal value of the measurement and the difference as a systematic uncertainty.
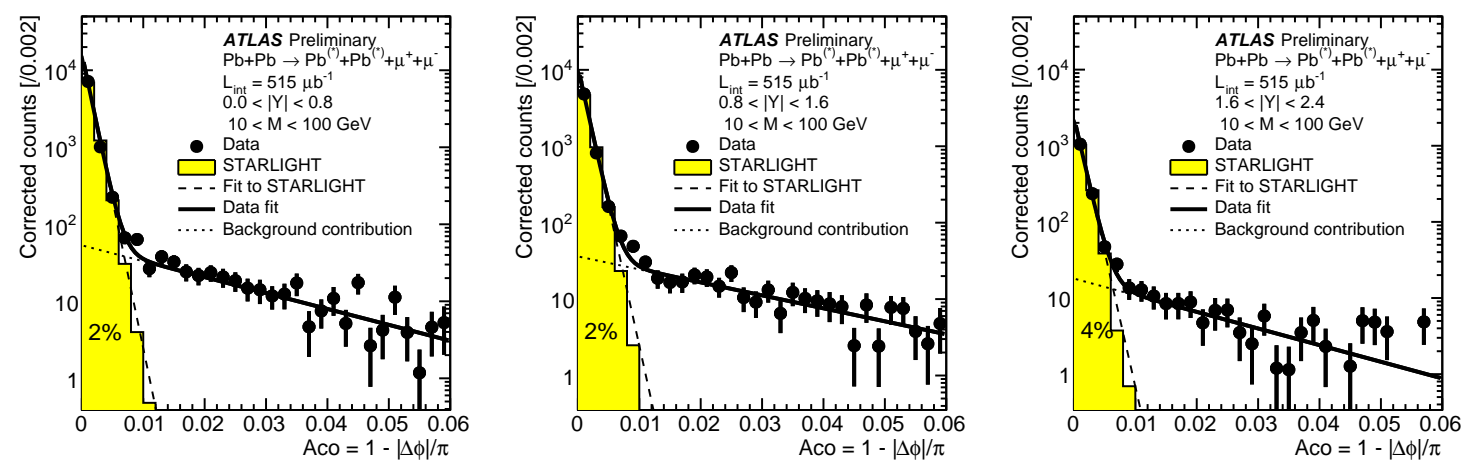

Figure 2: The acoplanarity distribution in data (black points) and Monte Carlo (yellow histogram) in different bins of rapidity of dimuon pair $Y$ [5].

The main contribution to the systematic uncertainty originates from the uncertainty related to the measurement of the luminosity and amounts to $7 \%$. 

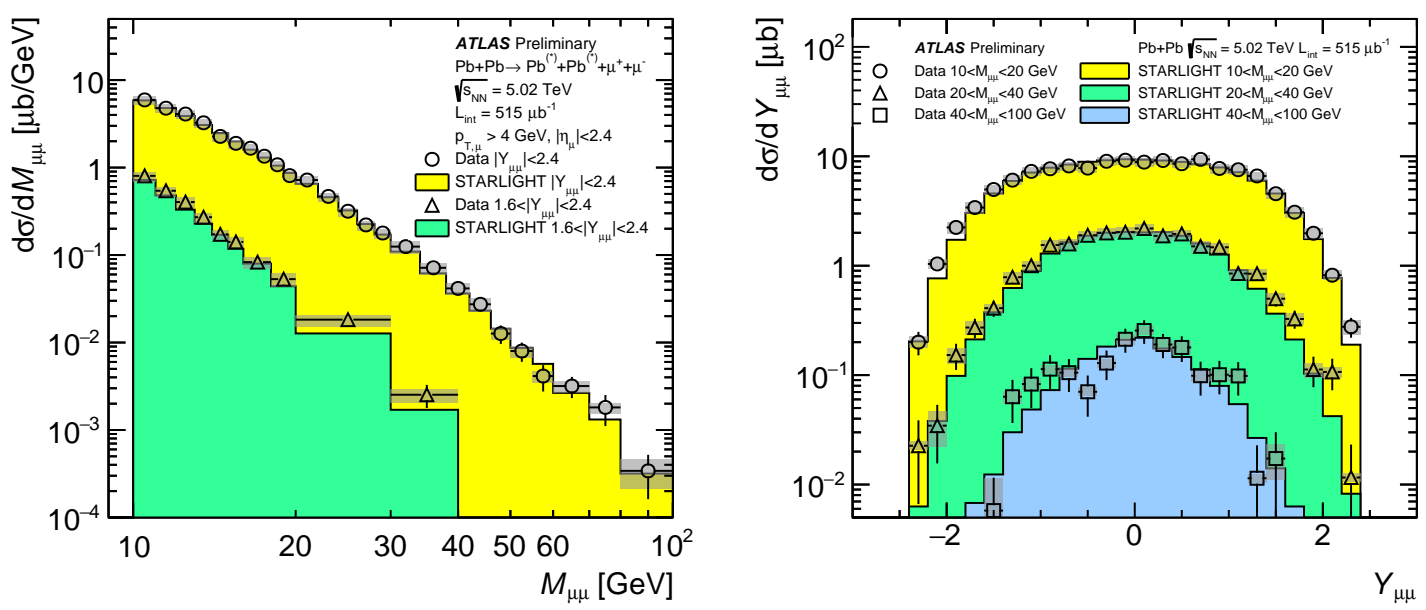

Figure 3: Cross-section for exclusive dimuon production in UPC, as a function of pair mass (left) and pair rapidity (right) [5].

The measured cross-section as functions of the dimuon mass $M_{\mu \mu}$ and rapidity $Y_{\mu \mu}$ (see Figure 3 ), as well as the total cross-section $\sigma=32.2 \pm 0.3$ (stat.) \pm 4.0 (syst.) $\mu \mathrm{b}$, are in good agreement with the STARlight MC $\left(\sigma_{\text {STARlight }}=31.6 \mu b\right)$.

\section{Search for light-by-light scattering}

The second reported measurement is the search for the process $\gamma \gamma \rightarrow \gamma \gamma$ known as the lightby-light scattering performed by ATLAS [7].

The motivation of this analysis is to measure for the first time in a direct way the photon on photon scattering. The previous indirect measurements were exploring the multi-photon BreitWheeler reaction $\left(\omega+n \omega_{0} \rightarrow e^{+} e^{-}\right)$[8], photon splitting [9] and Delbrück scattering [10].

A dedicated trigger for this analysis comprises the requirement of the total $E_{\mathrm{T}}$ in the calorimeter to be between 5 and $200 \mathrm{GeV}$, of no more than one hit in the inner MBTS and of less than 10 hits in the pixel detector.

As the offline selection, the events with two photons with $E_{\mathrm{T}}>3 \mathrm{GeV},|\eta|<2.37$ with no additional tracks from the interaction point, with the mass of the photon system $m_{\gamma \gamma}>6 \mathrm{GeV}$ and its transverse momentum $p_{\mathrm{T}}^{\gamma \gamma}<2 \mathrm{GeV}$ were selected. The additional cut on acoplanarity Aco $<0.01$ was applied to minimise the background from the Central Exclusive Production (CEP) of two photons $(g g \rightarrow \gamma \gamma)$.

The second significant source of background is constituted by the events with fake photons mainly originating from the dielectron pair production $\left(\gamma \gamma \rightarrow e^{+} e^{-}\right)$with electrons misidentified as photons.

The analysis of $\gamma \gamma \rightarrow \gamma \gamma$ process is exploring the kinematic region of very low transverse energy photons and as such requires special Photon IDentification (PID) as well as additional studies of photon reconstruction and its efficiency.

The PID was performed with use of three shower shape variables $E_{\text {ratio }}$ - the ratio of the energy difference associated with the largest and second largest energy deposits to the sum of these 
deposits in the first layer of EM calorimeter, $f_{1}$ - the fraction of energy reconstructed in the first layer with respect to the total energy of the cluster and $W_{\text {eta } 2}$ - the lateral width of the shower in the middle layer and optimised for low $p_{\mathrm{T}}$ photons with TMVA tool [11].

The PID, reconstruction, energy scale and resolution, as well as the trigger efficiency studies, were performed in data-driven way exploring the dilepton sample. For the reconstruction efficiency the $\gamma \gamma \rightarrow e^{+} e^{-}$events with one of the electrons emitting hard bremsstrahlung photon were used, in a similar fashion the PID was studied with the photons from final state radiation and the energy scale and resolution was tested with the electron energy deposits in the EM parts of the calorimeter. The uncertainties arising from the $\gamma$ reconstruction and PID are the dominating ones constituting $12 \%$ and $16 \%$ respectively.

The total of 13 events was selected in data with expected 7.3 of signal and 2.6 of background events. These data were used to derive the estimate of the significance of the observation of the light-by-light scattering at $4.4 \sigma$ with the profile likelihood method [12]. The predicted and measured log likelihood curve can be seen in Figure 4 on the left. The same events after applying the corrections and the subtraction of the background were used to calculate the cross-section in the phase space defined by the set of cuts on $p_{\mathrm{T}}^{\gamma}>3 \mathrm{GeV},\left|\eta^{\gamma}\right|<2.4, m_{\gamma \gamma}>6 \mathrm{GeV}, p_{\mathrm{T}}^{\gamma \gamma}<2 \mathrm{GeV}$, Aco $<0.01$. The measured cross-section $\sigma=70 \pm 24$ (stat.) \pm 17 (syst.) nb is in agreement within uncertainties with the Standard Model predictions 45 $\pm 9 \mathrm{nb}$ [13] and 49 $\pm 10 \mathrm{nb}$ [14].
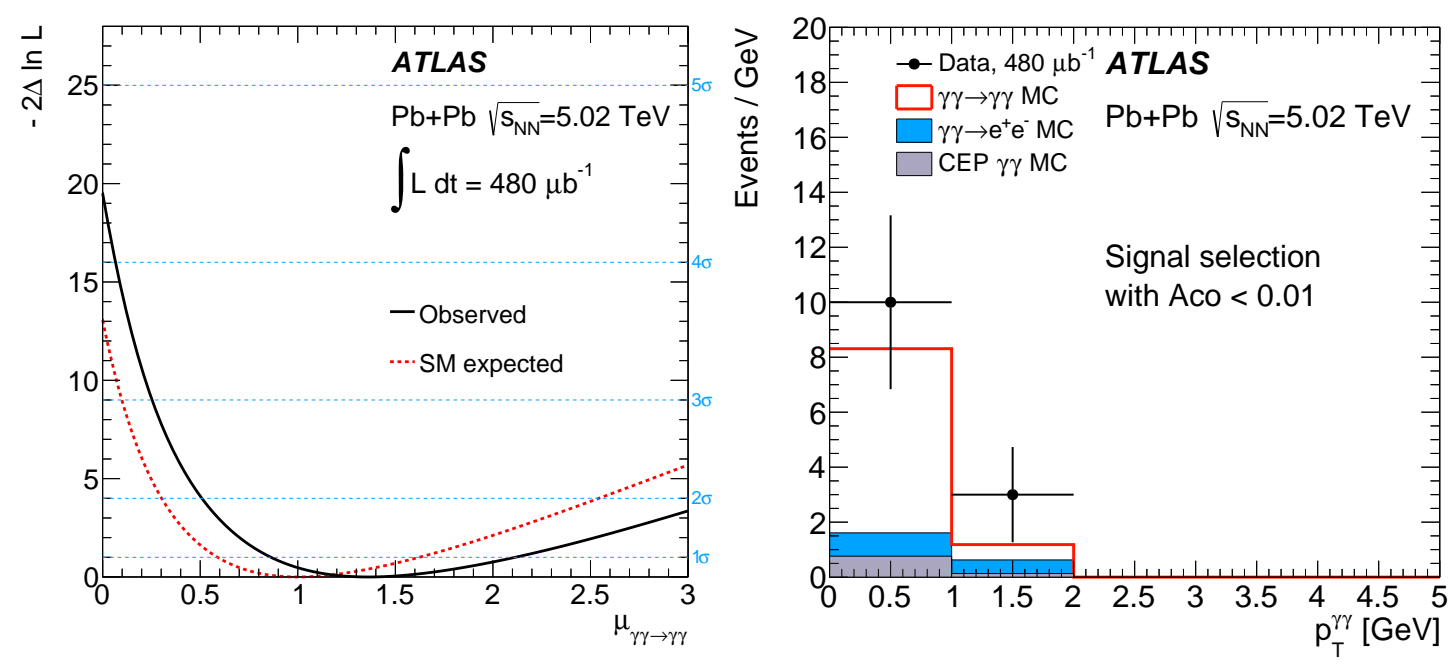

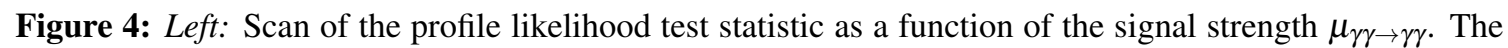
observed statistical significance for the background only hypothesis is $4.4 \sigma$, the expected one is $3.8 \sigma$ [7]. Right: Diphoton transverse momentum distribution. Points represent data while histograms are MC samples [7].

\section{Conclusions}

The ATLAS data from the ultra-peripheral collisions of the lead nuclei at $5.02 \mathrm{TeV}$ collected in 2015 with the total luminosity of $0.5 \mathrm{nb}^{-1}$ were used to investigate the $\gamma \gamma \rightarrow \mu \mu$ and $\gamma \gamma \rightarrow \gamma \gamma$ processes. The cross-sections for both of them were extracted and found to be in good agreement 
with the Standard Model predictions. The light-by-light scattering was observed for the first time with the significance of $4.4 \sigma$.

\section{Acknowledgements}

This work was supported in part by PL-Grid Infrastructure and by the National Science Center, Poland under grant DEC-2015/18/M/ST2/00087.

\section{References}

[1] E. Fermi, "On the Theory of Collisions between Atoms and Electrically Charged Particles," Nuovo Cim. 2 (1925) 143.

[2] C. F. von Weizsacker, "Radiation emitted in collisions of very fast electrons," Z. Phys. 88 (1934) 612.

[3] E. J. Williams, "Nature of the high-energy particles of penetrating radiation and status of ionization and radiation formulae," Phys. Rev. 45 (1934) 729.

[4] ATLAS Collaboration, "The ATLAS Experiment at the CERN Large Hadron Collider," JINST 3 (2008) S08003.

[5] ATLAS Collaboration, "Measurement of high-mass dimuon pairs from ultraperipheral lead-lead collisions at $\sqrt{s_{\mathrm{NN}}}=5.02 \mathrm{TeV}$ with the ATLAS detector at the LHC," ATLAS-CONF-2016-025 (2016), https://cds.cern.ch/record/2157689.

[6] S. R. Klein, J. Nystrand, J. Seger, Y. Gorbunov, and J. Butterworth, "STARlight: A Monte Carlo simulation program for ultra-peripheral collisions of relativistic ions," Comput. Phys. Commun. 212 (2017) 258, arXiv:1607.03838 [hep-ph].

[7] ATLAS Collaboration, "Evidence for light-by-light scattering in heavy-ion collisions with the ATLAS detector at the LHC," arXiv:1702.01625 [hep-ex].

[8] D. L. Burke et al., "Positron Production in Multiphoton Light-by-Light Scattering," Phys. Rev. Lett. 79 (1997) 1626.

[9] R. R. Wilson, "Scattering of 1.3 Mev Gamma-Rays by an Electric Field," Phys. Rev. 90 (1953) 720.

[10] Sh. Zh. Akhmadaliev et al., "Experimental investigation of high-energy photon splitting in atomic fields," Phys. Rev. Lett. 89 (2002) 061802, arXiv:hep-ex/0111084.

[11] A. Hoecker et al., "TMVA: Toolkit for Multivariate Data Analysis," PoS ACAT (2007) 040, arXiv:physics/0703039.

[12] G. Cowan et al., "Asymptotic formulae for likelihood-based tests of new physics," Eur. Phys. J. C71 (2011) 1554, [Erratum: Eur. Phys. J. C73 (2013) 2501], arXiv:1007.1727 [physics.data-an].

[13] D. d'Enterria, G. G. da Silveira, "Observing Light-by-Light Scattering at the Large Hadron Collider," Phys. Rev. Lett. 111 (2013) 080405, [Erratum: Phys. Rev. Lett. 116 (2016) 129901].

[14] M. Klusek-Gawenda, P. Lebiedowicz, A. Szczurek, "Light-by-light scattering in ultraperipheral Pb-Pb collisions at energies available at the CERN Large Hadron Collide," Phys. Rev. C93 (2016) 044907. 